\title{
Comparative Study between different Eigenspace-based Approaches for Face Recognition
}

\author{
Pablo Navarrete and Javier Ruiz-del-Solar \\ Department of Electrical Engineering, Universidad de Chile, CHILE \\ Email: \{pnavarre,jruizd\}@cec.uchile.cl
}

\begin{abstract}
Different eigenspace-based approaches have been proposed for the recognition of faces. They differ mostly in the kind of projection method been used and in the similarity matching criterion employed. The aim of this paper is to present a comparative study between some of these different approaches. This study considers theoretical aspects as well as simulations performed using a face database with a few number of classes.
\end{abstract}

\section{Introduction}

Among the most successful approaches used in face recognition we can mention eigenspace-based methods, which are mostly derived from the Eigenface-algorithm. These methods project the input faces onto a dimensional reduced space where the recognition is carried out, performing a holistic analysis of the faces. Different eigenspace-based approaches have been proposed. They differ mostly in the kind of projection/decomposition method been used and in the similarity matching criterion employed. The aim of this paper is to present a comparative study between some of these different approaches. The comparison considers the use of three different projection methods (Principal Component Analysis, Fisher Linear Discriminant and Evolutionary Pursuit) and five different similarity matching criteria (Euclidean-, Cosines- and Mahalanobis-distance, Self-Organizing Map and Fuzzy Feature Contrast). The pre-processing aspects of these approaches (normalization, illumination invariance, geometrical invariance, etc.) are not going to be addressed in this study. It should be noted that a previous comparative study that does not include the Fuzzy Feature Contrast method was presented in [4]. The mentioned methods are described in section 2 , and the comparative study is presented in section 3 .

\section{Eigenspace-based Approaches}

Eigenspace-based approaches approximate the face vectors (face images) with lower dimensional feature vectors. The main supposition behind this procedure is that the face space (given by the feature vectors) has a lower dimension than the image space (given by the number of pixels in the image), and that the recognition of the faces can be performed in this reduced space. These approaches consider an off-line phase or training, where the face database is created and the projection matrix, the one that achieve the dimensional reduction, is obtained from all the database face images. In the off-line phase are also calculated the mean face and the reduced 
representation of each database image. These representations are the ones to be used in the recognition process.

\subsection{General Approach}

Figure 1 shows the block diagram of a generic eigenspace-based face recognition system. A preprocessing module transforms the face image into a unitary vector and then performs a subtraction of the mean face $(\overline{\mathbf{x}})$. After that, the resulting vector, $\mathbf{x}$, is projected using the projection matrix $\mathbf{W} \in R^{N \times m}$ that depends on the eigenspace method been used (see section 2.2). This projection corresponds to a dimensional reduction of the input, starting with vectors $\mathbf{x}$ in $R^{N}$ (with $N$ the image vector dimension) and obtaining projected vectors $\mathbf{q}$ in $R^{m}$ with $m<N$ (usually $m<<N$ ). The Similarity Matching module compares the similarity of the reduced representation of the query face vector $\mathbf{q}$ with the reduced vectors $\mathbf{p}^{k} \in R^{m}$ that represent the faces in the database. By using a given criterion of similarity (see section 2.3), this module determines the most similar vector $\mathbf{p}^{k}$ in the database. The class of this vector is the result of the recognition process, i.e. the identity of the face. In addition, a Rejection System for unknown faces is used if the similarity matching measure is not good enough (see description in [1]).

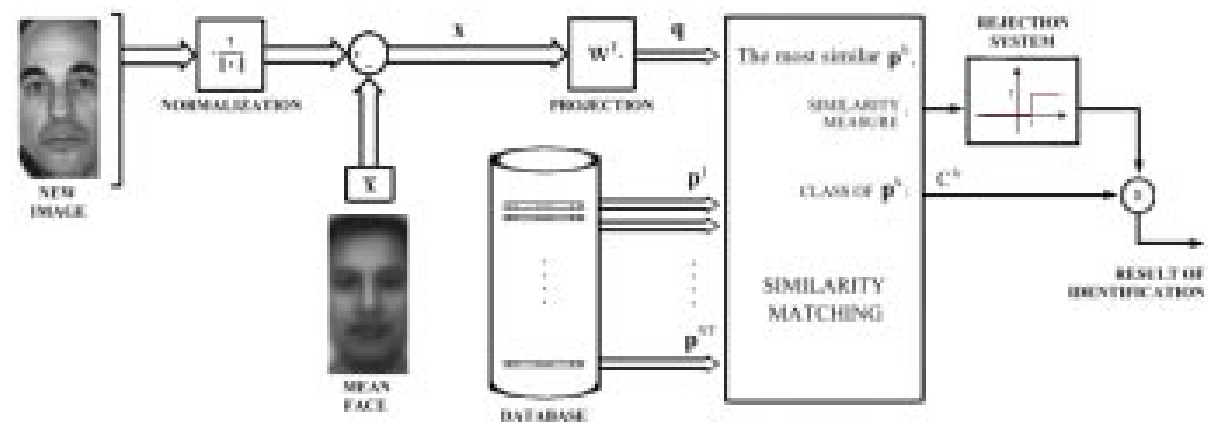

Fig. 1. Block diagram of a given eigen-space face recognition system.

\subsection{Projection/Decomposition Methods}

Principal Components Analysis - PCA

PCA is a general method to identify the principal differences between signals and after that to make a dimensional reduction of them. Let $\mathbf{X}=\left[\left(\mathbf{x}^{1}-\overline{\mathbf{x}}\right)\left(\mathbf{x}^{2}-\overline{\mathbf{x}}\right) \cdots\left(\mathbf{x}^{N T}-\overline{\mathbf{x}}\right)\right]$ be the matrix of the normalized training vectors. $\mathbf{x}^{j}$ represents the normalized $j$ image vector, $\mathbf{x}$ is the mean face image and $N T$ is the number of training images. Then, $\mathbf{R}=\mathbf{X} \mathbf{X}^{\mathrm{T}}$ will be the correlation matrix estimator. The eigenvectors of $\mathbf{R}$ represent a special basis in the image space, and the eigenvalues are the projection variance on each of this axes (the Eigenfaces). Therefore PCA will chose only the eigenvectors of $\mathbf{R}$ associated with the higher 
variance and in this way will reduce the dimension of the training images. Also PCA give us the projection matrix $\mathbf{W} \in R^{N \times m}$ for reducing every image that follows the same statistical pattern. Computational aspects of the implementation of this method are explained in [4].

\section{Fisher Linear Discriminant - FLD}

FLD searches for the projection axes on which the face images of different classes are far from each other, and at the same time where the face images of the same class are close from each other. In a similar way of PCA using the $\mathbf{R}$ matrix, FLD uses two scatter matrices, $\mathbf{S}_{b}$ and $\mathbf{S}_{w}$, for representing the separation between the individual class means respect to the global mean face, and the separation between vectors of each class respect to their own class mean, respectively:

$\mathbf{S}_{b}=\sum_{i=1}^{N C} \mathrm{P}\left(C_{i}\right)\left(\mathbf{m}^{(i)}-\mathbf{m}\right)\left(\mathbf{m}^{(i)}-\mathbf{m}\right)^{\mathrm{T}} ; \mathbf{S}_{w}=\sum_{i=1}^{N C} \mathrm{P}\left(C_{i}\right) \mathrm{E}\left[\left(\mathbf{x}^{(i)}-\mathbf{m}^{(i)}\right)\left(\mathbf{x}^{(i)}-\mathbf{m}^{(i)}\right)^{\mathrm{T}}\right]$

where $\mathbf{m}$ is the global mean vector, $\mathrm{P}\left(C_{i}\right)$ are the occurrence probabilities associated to each class $C_{i}, \mathbf{m}^{(i)}$ are the average vectors of $C_{i}$, and $\mathbf{x}^{(i)}$ are the vectors associated to $C_{i}$. The maximization of the between class scatter and the minimization of the within class scatter is performed by solving the general eigensystem $\mathbf{S}_{b} \mathbf{w}^{k}=\lambda_{k} \mathbf{S}_{w} \mathbf{w}^{k}$. The resulting non-orthonormal base represents the projection matrix $\mathbf{W} \in R^{N \times m}$, where the $m$ rows are the general eigenvectors associated with the largest general eigenvalues (Fisher Parameters [4]). To solve the problem of the large size of the scatter matrices, PCA is applied before FLD. In this way we are also solving the problem of singularity for $\mathbf{S}_{w}$.

\section{Evolutionary Pursuit - EP}

EP, originally proposed in [3], searches for the best set of projection axes in order to maximize a fitness function that measures, at the same time, the classification accuracy and generalization ability of the system. Because the dimension of the solution-space of this problem is very large, it is solved using Genetic Algorithms. In order to obtain the EP-faces an initial dimensional reduction is first performed using PCA, and then a Whitening Transformation is applied (equivalent to a Mahalanobis metric system, see 2.3). In the Whitened-PCA space are performed several rotations between pair of axes and then a subset of them is selected. Each rotation is coded using a chromosome representation. In this representation each chromosome represents a certain projection system. To evaluate this system the following fitness function is used:

$$
\zeta\left(\alpha_{k}, a_{i}\right)=\zeta_{a}\left(\alpha_{k}, a_{i}\right)+\lambda \zeta_{s}\left(\alpha_{k}, a_{i}\right),
$$

where $\zeta_{a}\left(\alpha_{k}, a_{i}\right)$ measures the accuracy, $\zeta_{s}\left(\alpha_{k}, a_{i}\right)$ measures the generalization ability, and $\lambda$ is a positive constant (see definitions in [3]). 


\subsection{Similarity Matching Methods}

\section{Euclidean Distance}

$$
d(\mathbf{x}, \mathbf{y})=\sqrt{(\mathbf{x}-\mathbf{y})^{\mathrm{T}}(\mathbf{x}-\mathbf{y})} .
$$

\section{Cosine Distance}

$$
\cos (\mathbf{x}, \mathbf{y})=\frac{\mathbf{x}^{\mathrm{T}} \mathbf{y}}{\|\mathbf{x}\|\|\mathbf{y}\|}
$$

\section{Mahalanobis Distance}

$$
d(\mathbf{x}, \mathbf{y})=(\mathbf{x}-\mathbf{y})^{\mathrm{T}} \mathbf{R}^{-1}(\mathbf{x}-\mathbf{y}) ; \quad \mathbf{R} \text { : correlation matrix. }
$$

From a geometrical point of view this distance has a scaling effect in the image space. Taking into consideration the face image subset, directions in which a greater variance exist are compressed and directions in which a smaller variance exist are expanded. It can be proved that in the PCA space the Mahalanobis distance is equivalent to the Euclidean distance, weighting each component by the inverse correspondent eigenvalue (see demonstration in [4]), and it is often called Whitening (PCA) Transformation.

\section{SOM Clustering}

Self-Organizing Maps (SOMs) are used as associative networks to match the projected query face with the corresponding projected database faces. The use of a SOM to implement this module improves the generalization ability of the system. The SOM approach uses reference vectors $\mathbf{m}_{i}$ to approximate the probability distribution of the faces in a 2D map [2]. In the training phase of the SOM a clustering of the reduced face vectors is carried out. Thereafter the SOM is transformed in an associative network by labeling all its nodes. Both procedures are explained in [4].

Fuzzy Feature Contrast - FFC

$$
S(\mathbf{x}, \mathbf{y})=\sum_{i=1}^{m} \min \left\{\mu_{i}(\mathbf{x}), \mu_{i}(\mathbf{y})\right\}-\alpha \sum_{i=1}^{m} \max \left\{\mu_{i}(\mathbf{x})-\mu_{i}(\mathbf{y}), 0\right\}-\beta \sum_{i=1}^{m} \max \left\{\mu_{i}(\mathbf{y})-\mu_{i}(\mathbf{x}), 0\right\}
$$

where $\mu_{i}(\mathbf{x})$ is a membership function associated with the i-component of vector $\mathbf{x} \in R^{m}$. This similarity measure, originaly proposed in [5], is a fuzzy implementation of the Feature Contrast model from Tversky. The first sum measure the common features (intersection) and the others represent the distinctive features (difference in the two possible ways). The positive parameters $\alpha$ and $\beta$ adjust the contrast of the three kind of features. By chosing $\alpha \neq \beta$ it is possible to introduce asymetries between 
the subject-referent comparison. This model considers that all the features are independent, and that can be assumed in PCA and WPCA, but not in FLD and EP. In our implementation we normalize each feature of PCA (in WPCA it is not necessary) and we chosed $\mu_{i(\mathbf{x})}$ linear between -1 and 1 , with $x_{i}$ normalized.

\section{Comparison among the approaches}

In order to test the described methods we have made several simulations based in the Yale University - Face Image Database. We use 150 images of 15 different classes. Then we preprocessed the images by masking them in windows of $100 \times 200$ pixels placing the several face features in the same relative places. In table 1 we show the results of several simulations using different kind of representations and similarity matching methods. For each simulation we used a fixed number of training images, using the same type of images per class, according with the Yale database specification. In order to obtain representative results we take the average of 20 different set of images for each fixed number of training images. All the images not used for training are used for testing.

We can see that the best models always are obtained with the Fisher representation, and the difference against the other representations decrease when the number of training images per class decrease, showing that the FLD discrimination ability strongly depends on the number of training images per class. The best results are almost always obtained with FLD- cosine. The systems that seem to be as efficient as FLD-cosine are SOM and Withening-cosine.

The best results using FFC were obtained employing an asymmetric subject-referent comparison: $\alpha=0.5 \beta=5$. This means that in the question "how is the subject face similar to the referent face?" the answer focus more on the features of the referent (the unknown face). The generalization ability of the systems is not well measured in our simulations because the number of selected axes is about the same of the number of classes (15). That affects the FLD representation method as well as the FFC and SOM similarity matching methods. For this reason in future works we want to perform our comparative study on a larger database, like FERET. We think that this will improve the relative recognition ability of the methods being affected for the small number of classes.

Another important issue is the computational cost of the training processes. In PCA this computational cost is mainly due to the process of determining $\mathbf{R}$, $O\left(N T^{2} \cdot N\right)$, and solving the eigensystem, $O\left(N T^{3}\right)$. If we suppose that the number of training images $N T$ is much smaller than the number of pixels per image $N$, then the computational cost of PCA is just the cost of determining $\mathbf{R}, O\left(N T^{2} \cdot N\right)$. In our implementation of FLD we requires previously the computation of PCA to reduce the vectors dimension to $m_{1}\left(m_{1}<N T\right)$, and the additional cost is due to the process of determining the scatter matrices, $O\left(m_{1}^{2} \cdot N C\right)$, and solving the general eigensystem, $O\left(m_{1}^{3}\right)$. Nevertheless the additional cost in FLD is usually much smaller than the PCA initial cost. Finally EP requires much more computations because this process must iterate until a given criterion is accomplished. The computational cost of on-line operation is mainly given by the comparisons with database vectors, $O(N T \cdot m)$, 
except when the SOM-based similarity measure is used, $O(($ number of nodes $) \cdot m)$. The numerical stability for the different methods depends mostly of the numerical algorithms used for solving eigensystems. Either in PCA or FLD this is not a critical problem because always involves the management of symmetric matrices.

Table 1. Mean recognition rates using different numbers of training images per class, and taking the average of 20 different training sets. The small numbers are the standard deviation of each recognition rate.

\begin{tabular}{|c|c|c|c|c|c|c|c|c|c|c|}
\hline & \multirow[b]{2}{*}{$\mathrm{im} / \mathrm{chss}$} & \multirow[b]{2}{*}{ axes } & \multirow[b]{2}{*}{ Euclidean } & \multirow[b]{2}{*}{$\cos (?)$} & \multirow[b]{2}{*}{ SOM } & \multirow[b]{2}{*}{ FFC } & \multirow{2}{*}{\begin{tabular}{|c|} 
Whitening \\
Euclidean
\end{tabular}} & \multirow{2}{*}{$\begin{array}{l}\text { Whitening } \\
\cos (?)\end{array}$} & \multirow{2}{*}{$\begin{array}{l}\text { Whitening } \\
\text { SOM }\end{array}$} & \multirow{2}{*}{$\begin{array}{c}\text { Whitening } \\
\text { FFC }\end{array}$} \\
\hline & & & & & & & & & & \\
\hline PCA & \multirow{3}{*}{6} & 56 & $\begin{array}{c}87.9 \\
6.2 \\
\end{array}$ & $\begin{array}{c}86.0 \\
6.8 \\
\end{array}$ & $\begin{array}{c}84.6 \\
7.0 \\
\end{array}$ & $\begin{array}{c}77.1 \\
101 \\
\end{array}$ & $\begin{array}{c}64.7 \\
9.4 \\
\end{array}$ & $\begin{array}{c}79.3 \\
11.6 \\
\end{array}$ & $\begin{array}{c}64.7 \\
10.5 \\
\end{array}$ & $\begin{array}{c}77.1 \\
101 \\
\end{array}$ \\
\hline FISHER & & 17 & $\begin{array}{c}91.5 \\
6.6 \\
\end{array}$ & $\begin{array}{c}91.6 \\
6.5 \\
\end{array}$ & $\begin{array}{c}90.3 \\
6.7 \\
\end{array}$ & $\begin{array}{c}83.9 \\
9.3 \\
\end{array}$ & $\begin{array}{c}91.9 \\
58\end{array}$ & $\begin{array}{c}92.6 \\
5.6\end{array}$ & $\begin{array}{c}92.1 \\
6.2 \\
\end{array}$ & $\begin{array}{c}85.6 \\
83 \\
\end{array}$ \\
\hline E.P. & & 16 & $\begin{array}{c}81.2 \\
9.0 \\
\end{array}$ & $\begin{array}{c}85.3 \\
8.7 \\
\end{array}$ & $\begin{array}{c}83.7 \\
9.8 \\
\end{array}$ & $\begin{array}{c}77.2 \\
8.0 \\
\end{array}$ & - & - & - & - \\
\hline PCA & \multirow{3}{*}{5} & 34 & $\begin{array}{c}88.7 \\
3.8 \\
\end{array}$ & $\begin{array}{c}87.1 \\
4.1 \\
\end{array}$ & $\begin{array}{c}86.0 \\
5.1 \\
\end{array}$ & $\begin{array}{c}78.5 \\
8.1 \\
\end{array}$ & $\begin{array}{c}69.5 \\
89 . \\
\end{array}$ & $\begin{array}{c}83.2 \\
9.0 \\
\end{array}$ & $\begin{array}{c}66.1 \\
10.5 \\
\end{array}$ & $\begin{array}{c}78.5 \\
8.1 \\
\end{array}$ \\
\hline FISHER & & 15 & $\begin{array}{c}92.2 \\
5.7\end{array}$ & $\begin{array}{c}91.7 \\
6.2 \\
\end{array}$ & $\begin{array}{c}90.3 \\
6.4 \\
\end{array}$ & $\begin{array}{c}85.1 \\
9.1\end{array}$ & $\begin{array}{c}92.3 \\
4.7\end{array}$ & $\begin{array}{c}92.4 \\
5.7\end{array}$ & $\begin{array}{c}92.1 \\
5.3 \\
\end{array}$ & $\begin{array}{c}85.4 \\
85 \\
\end{array}$ \\
\hline E.P. & & 13 & $\begin{array}{c}84.1 \\
5.7 \\
\end{array}$ & $\begin{array}{c}87.7 \\
6.6 \\
\end{array}$ & $\begin{array}{c}86.7 \\
7.6 \\
\end{array}$ & $\begin{array}{c}78.7 \\
6.8 \\
\end{array}$ & - & - & - & - \\
\hline PCA & \multirow{3}{*}{4} & 46 & $\begin{array}{c}87.3 \\
3.9 \\
\end{array}$ & $\begin{array}{c}86.7 \\
3.9 \\
\end{array}$ & $\begin{array}{c}84.8 \\
3.6 \\
\end{array}$ & $\begin{array}{c}77.6 \\
5.2 \\
\end{array}$ & $\begin{array}{c}72.9 \\
55 \\
\end{array}$ & $\begin{array}{c}84.4 \\
5.6 \\
\end{array}$ & $\begin{array}{c}66.7 \\
6.5 \\
\end{array}$ & $\begin{array}{c}77.6 \\
52 \\
\end{array}$ \\
\hline FISHER & & 18 & $\begin{array}{c}90.3 \\
4.5 \\
\end{array}$ & $\begin{array}{c}91.1 \\
5.0 \\
\end{array}$ & $\begin{array}{c}90.3 \\
4.4 \\
\end{array}$ & $\begin{array}{c}84.4 \\
5.9 \\
\end{array}$ & $\begin{array}{c}90.4 \\
42 \\
\end{array}$ & $\begin{array}{c}91.0 \\
4.4 \\
\end{array}$ & $\begin{array}{c}90.1 \\
4.7 \\
\end{array}$ & $\begin{array}{c}82.9 \\
5.7 \\
\end{array}$ \\
\hline E.P. & & 18 & $\begin{array}{c}83.6 \\
4.6 \\
\end{array}$ & $\begin{array}{c}86.9 \\
4.7 \\
\end{array}$ & $\begin{array}{c}85.0 \\
5.0 \\
\end{array}$ & $\begin{array}{c}74.7 \\
6.0 \\
\end{array}$ & - & - & - & - \\
\hline PCA & \multirow{3}{*}{3} & 35 & $\begin{array}{c}86.6 \\
4.0 \\
\end{array}$ & $\begin{array}{c}85.4 \\
3.9 \\
\end{array}$ & $\begin{array}{c}82.0 \\
5.6\end{array}$ & $\begin{array}{c}77.9 \\
4.6 \\
\end{array}$ & $\begin{array}{c}75.0 \\
56 \\
\end{array}$ & $\begin{array}{c}84.8 \\
5.4\end{array}$ & $\begin{array}{c}67.4 \\
6.9 \\
\end{array}$ & $\begin{array}{c}77.9 \\
4.6 \\
\end{array}$ \\
\hline FISHER & & 15 & $\begin{array}{c}89.0 \\
3.6 \\
\end{array}$ & $\begin{array}{c}90.4 \\
4.0 \\
\end{array}$ & $\begin{array}{c}87.4 \\
4.0 \\
\end{array}$ & $\begin{array}{c}80.7 \\
6.3 \\
\end{array}$ & $\begin{array}{c}88.9 \\
3.1 \\
\end{array}$ & $\begin{array}{c}89.9 \\
3.9 \\
\end{array}$ & $\begin{array}{c}88.7 \\
3.9 \\
\end{array}$ & $\begin{array}{c}81.5 \\
3.4 \\
\end{array}$ \\
\hline E.P. & & 14 & $\begin{array}{c}81.1 \\
4.3 \\
\end{array}$ & $\begin{array}{c}86.9 \\
3.7 \\
\end{array}$ & $\begin{array}{c}82.5 \\
3.7 \\
\end{array}$ & $\begin{array}{c}75.9 \\
4.4 \\
\end{array}$ & - & - & - & - \\
\hline$\overline{P \text { PCA }}$ & \multirow{3}{*}{2} & 26 & $\begin{array}{c}82.7 \\
5.9 \\
\end{array}$ & $\begin{array}{c}80.8 \\
5.9 \\
\end{array}$ & $\begin{array}{c}76.2 \\
7.9 \\
\end{array}$ & $\begin{array}{c}71.1 \\
5.9 \\
\end{array}$ & $\begin{array}{c}75.6 \\
49 \\
\end{array}$ & $\begin{array}{c}82.1 \\
4.6 \\
\end{array}$ & $\begin{array}{c}60.8 \\
7.3 \\
\end{array}$ & $\begin{array}{c}71.1 \\
59 \\
\end{array}$ \\
\hline FISHER & & 15 & $\begin{array}{c}81.5 \\
5.6\end{array}$ & $\begin{array}{c}82.2 \\
5.8\end{array}$ & $\begin{array}{c}79.4 \\
5.8 \\
\end{array}$ & $\begin{array}{c}69.3 \\
8.6 \\
\end{array}$ & $\begin{array}{c}80.7 \\
4.7 \\
\end{array}$ & $\begin{array}{c}82.8 \\
4.9 \\
\end{array}$ & $\begin{array}{c}78.8 \\
5.8 \\
\end{array}$ & $\begin{array}{c}73.6 \\
62 \\
\end{array}$ \\
\hline E.P. & & 14 & $\begin{array}{c}77.8 \\
5.6 \\
\end{array}$ & $\begin{array}{c}81.2 \\
5.3\end{array}$ & $\begin{array}{c}76.0 \\
7.3 \\
\end{array}$ & $\begin{array}{c}70.0 \\
7.4 \\
\end{array}$ & - & - & - & \\
\hline
\end{tabular}

\section{Acknowledgements}

This research was supported by the DID (U. de Chile) under Project ENL-2001/11 and by the join "Program of Scientific Cooperation" of CONICYT (Chile) and BMBF (Germany).

\section{References}

1. Golfarelli M., Maio D., and Maltoni D., "On the Error-Reject Trade-Off in Biometric Verification Systems", IEEE Trans. Pattern Analysis and Machine Intelligence, vol. 19, no. 7, pp. 786-796, July 1997.

2. Kohonen T., "Self-Organized Maps", 1997. 
3. Liu C., and Wechsler H., "Evolutionary Pursuit and Its Application to Face Recognition", IEEE Trans. Pattern Analysis and Machine Intelligence, vol. 22, no. 6, pp. 570-582, June 2000.

4. Navarrete P., and Ruiz del Solar J., "Eigenspace-based Recognition of Faces: Comparisons and a new Approach", Proc. of the Int. Conf. on Image Analysis and Processing ICIAP 2001, pp. 42-47, Sept. 26-28, Palermo, Italy.

5. Santini S., and Jain R., "Similarity Measures", IEEE Trans. Pattern Analysis and Machine Intelligence, vol. 21, no. 9, pp. 871-883, September 1999. 